\title{
A Survey on Clinical Research Training Status and Needs in Public Hospitals from Shenzhen
}

\author{
Ping $\mathrm{Ji}^{1}$, Haibo Wang ${ }^{2}$, Chao Zhang ${ }^{1}$, Min $\mathrm{Liu}^{3}$, Liping Zhou ${ }^{3}$, Ping Xiao ${ }^{1}$, Yanfang Wang ${ }^{2}$, Yangfeng $\mathrm{Wu}^{2}$ \\ ${ }^{1}$ Shenzhen- Peking University - The Hongkong University of Science \& Technology Medical Center, Shenzhen, China \\ ${ }^{2}$ Peking University Clinical Research Institute, Beijing, China \\ ${ }^{3}$ Health and Family Planning Commission of Shenzhen, Shenzhen, China \\ Correspondence: Ping Ji, Shenzhen Peking University - Hongkong University of Science \& Technology Medical Center, \\ Shenzhen, China.
}

Received: April 28, 2017

Accepted: June 20, 2017 Online Published: June 20, 2017

doi:10.11114/jets.v5i7.2501

URL: https://doi.org/10.11114/jets.v5i7.2501

\begin{abstract}
Objective: To obtain information on the current clinical research training status and evaluate the training needs comprehensively for medical staff in hospitals.

Methods: This survey was initiated and conducted by the Health and Family Planning Commission of Shenzhen in conjunction with the Peking University Clinical Research Institute (Shenzhen) from Sep 2016 through Nov 2016. It was planned that no less than $10 \%$ of the total staff from each participating hospital were invited to complete the survey. All participants filled out the questionnaire anonymously and voluntarily.

Results: A total of 644 subjects from 12 hospitals completed the survey with the response rate of $28.7 \%$. The majority of respondents (80\%) have attended training related to clinical research knowledge and skills, however, medical research ethical aspects and statistics knowledge have been provided only for $40 \%$ and $27 \%$ of respondents, respectively. With regard to preference on training subjects and contents, Protocol design and development (74\%) is ranked first, followed by the data analysis and summary (59\%) among those researchers from medical professions. Project management and quality control course are mostly demanded for technicians and nurses (53\%). Unpredictably, no more than $40 \%$ of the respondents consider that it is necessary to receive "Regulation and Research ethics" course even for those Principal investigators.
\end{abstract}

Conclusions: Our survey results indicated that training subjects on protocol design and research ethics are urgently needed and relatively inadequate in China. An appropriate clinical research competency training model should be developed and provided for hospital staffs in China.

Keywords: clinical research, training, hospital staff, ethical training

\section{Introduction}

The importance of clinical research studies has been recognized gradually since it was stated that clinical research was the neck of the scientific bottle through which all scientific developments in biomedicine must flow before they can be of real-world benefit to the public. It has been advocated that the ability of medical school and hospitals to conduct clinical research properly and train responsible investigators must be supported and strengthened.

Chinese laws and guidelines for research have been rapidly produced within the last decade. The National Health and Family Planning Commission of China, together with Ministry of Science and Technology and other key regulators, has advocated to enhance clinical research capability building in 2016. As China's first special economic zone, Shenzhen has created an innovative spirit, namely as Chinese Silicon Valley. For instance, it plays a leading role in medical device industry in China with more than 1000 medical device companies. However, clinical research productivity is relatively low in Shenzhen. To keep pace with national clinical research and its rapid growth, "Shenzhen Clinical Research Program and Center" has been newly launched by local governors and hospitals. There are particular challenges, especially the lack of qualified and well-trained personnel and a professional support platform for designing and conducting the clinical research in order to meet the scientific, ethical, feasible and multi-fields management requirements. Zhang reported that researchers in state certified site of Traditional Chinese Medicine need to enhance the 
training on systematic knowledge of scientific methodology(Zhang et al.,2010).Deficient clinical research training courses during the medical education are provided both for undergraduate and graduate students in China (Hu et al., 2011). Wang and Liu stated that the slow development of clinical research in China is due to the lack of systematic training in clinical research (Wang and Liu, 2013). Zhang conducted a study to investigate the basic knowledge on study design among medical doctors and indicated that principles of clinical research were unclear (Zhang et al., 2015). Thus, demands on research talents training are rapidly increased.

Our question was if available training modes and resources were meeting the needs of the medical staff in hospitals in Shenzhen. We aimed to obtain information on the current clinical research training status and to evaluate the training needs comprehensively for medical staff in hospitals. Therefore, learning requirements from the perspective of doctors and nurses may integrate into the training program in a systematic and standardized manner.

This survey was initiated and conducted by the Health and Family Planning Commission of Shenzhen in conjunction with the Peking University Clinical Research Institute (Shenzhen).

\section{Methods}

\subsection{Study Design}

The cross-sectional study was conducted from Sep 2016 through Nov 2016 in Shenzhen. With approval of from the Peking University Biomedical IRB. We conducted a web-based survey among 19 public first tier hospitals in terms of hospital classification in China. The enrollment criteria of subjects included: 1) medical doctors, nurses, Laboratory Medicine and research faculty who have worked longer than 1 year in their current hospital;2) their willingness to participate.

The questionnaire consisted of the following information: 1) Personal characteristics, including working department, job categories, education background, research experience; 2) training status and requirements, including training subjects, contents, length, type and location; 3) open-ended questions regarding to the elements of the course they valued the most, and areas requiring improvement. It took 10-15 minutes to complete.

\subsection{Sampling Method}

The 19 hospitals were recommended by the Health and Family Planning Commission of Shenzhen according to the previous record for conducting research from 23 public first tier hospitals in Shenzhen. We aimed to randomly select no less than $10 \%$ of the total number of staffs in each hospital.

\subsection{Survey Procedure and Data Collection}

After receiving the eligible staff coding list from each hospital, we randomly selected subjects and send the selected staff code list back to the hospital. The personnel from research management office of hospital identified the individual staff and obtain their contact information. All the selected staff were invited to complete the web-based survey by sending the reminding text message for at least twice. Those participators completed the online questionnaire anonymously. There were respondents in 2 hospitals who filled the survey in paper and collected by the hospital research management office due to the inconvenience to get access to the internet in the hospital. Those paper-copied questionnaire were entered to the database by team member.

\subsection{Statistic Analysis}

Statistical tests were performed using SAS ${ }^{\mathrm{TM}} 9.4$ software (SAS Institute Inc., Cary, NC, USA). Results were presented as mean \pm standard deviation (SD) for continuous variables and frequency (proportion) for categorical variables. The significance of differences among different job categories was evaluated using chi-square tests for categorical variables and the Kruskal-Wallis test for continuous variables. All tests were two-sided and p-value $<0.05$ was considered the cut-off level for statistical significance for all analyses.

\section{Results}

\subsection{Subject's Characteristics}

A total of 644 subjects from 12 hospitals completed the survey. The overall response rate was only $28.7 \%$. Among that number, $54.2 \%(\mathrm{n}=349)$ have attended the patient-oriented research. Table 1 shows the characteristics of subjects. 
Table 1. Subjects' characteristics

\begin{tabular}{lll}
\hline Characteristics & & $\mathrm{N}(\%)$ \\
\hline Professional Title & Junior & $284(44.1)$ \\
& Middle & $223(34.6)$ \\
Job Categories & Senior & $137(21.3)$ \\
& Medical doctors/research faculty & $286(44.4)$ \\
Laboratory Medicine & $103(16.0)$ \\
Negree & Nurse & $255(39.6)$ \\
& Bachelor & $274(42.6)$ \\
Research Role & Master & $129(20.0)$ \\
& Doctor & $41(6.4)$ \\
& Others & $200(31.1)$ \\
& Principle Investigator & $184(28.6)$ \\
& Coordinator & $26(4.0)$ \\
& Sub-Investigator & $60(9.3)$ \\
& Team member & $374(58.1)$ \\
& Bio-engineer & $60(9.3)$ \\
& Others & $88(13.7)$ \\
\hline
\end{tabular}

\subsection{Training Status}

Regarding training methods and resources, academic conferences and training courses accounted for $79.2 \%$ and $78 \%$, respectively. In total, the majority of respondents $(79.5 \%, \mathrm{n}=512)$ have attended training for clinical research knowledge and skills. However, medical research ethical aspects and statistics knowledge have been provided only for $40.4 \%$ $(n=260)$ and $27.0 \%(n=174)$ of the respondents, respectively. Training status based on different professional titles is shown in Table 2.

Table 2. Training status by professional title

\begin{tabular}{|c|c|c|c|c|c|c|}
\hline & & $\begin{array}{l}\text { Junior } \\
(\mathrm{N}=284) \\
\mathrm{N}(\%)\end{array}$ & $\begin{array}{l}\text { Middle } \\
(\mathrm{N}=223) \\
\mathrm{N}(\%)\end{array}$ & $\begin{array}{l}\text { Senior } \\
(\mathrm{N}=137) \\
\mathrm{N}(\%)\end{array}$ & $\begin{array}{l}\text { Total } \\
(\mathrm{N}=644) \\
\mathrm{N}(\%)\end{array}$ & P-value \\
\hline \multirow{5}{*}{$\begin{array}{l}\text { Subjects and } \\
\text { Contents }\end{array}$} & clinical research & & & & & 0.0005 \\
\hline & $\begin{array}{l}\text { knowledge and } \\
\text { skills }\end{array}$ & $214(75.4)$ & $173(77.6)$ & $125(91.2)$ & $512(79.5)$ & \\
\hline & $\begin{array}{l}\text { statistics } \\
\text { knowledge }\end{array}$ & $56(19.7)$ & $61(27.4)$ & $58(42.3)$ & $175(27.2)$ & $<.0001$ \\
\hline & $\begin{array}{l}\text { medical research } \\
\text { ethic }\end{array}$ & $109(38.4)$ & $87(39.0)$ & $64(46.7)$ & $260(40.4)$ & 0.2312 \\
\hline & Others & $23(8.1)$ & $23(10.3)$ & $12(8.8)$ & $58(9.0)$ & 0.6835 \\
\hline \multicolumn{2}{|c|}{ Length of training (Days) } & $3.0 \pm 13.0$ & $5.0 \pm 57.0$ & $30.0 \pm 177.0$ & $4.0 \pm 53.0$ & $<.0001$ \\
\hline \multirow[t]{4}{*}{ Location } & Oversea & $3(1.1)$ & $8(3.6)$ & $33(24.1)$ & $44(6.8)$ & $<.0001$ \\
\hline & $\begin{array}{ll}\text { Outside } & \text { of } \\
\text { Shenzhen } & \end{array}$ & $38(13.4)$ & $74(33.2)$ & $83(60.6)$ & $195(30.3)$ & $<.0001$ \\
\hline & Within Shenzhen & $191(67.3)$ & $163(73.1)$ & $100(73.0)$ & $454(70.5)$ & 0.2767 \\
\hline & Working hospital & $190(66.9)$ & $142(63.7)$ & $72(52.6)$ & $404(62.7)$ & 0.0160 \\
\hline
\end{tabular}

In terms of "Professional Title", it was shown that there were statistically significant more Seniors who attended training for clinical research knowledge and skills and statistics knowledge than juniors and middles. However, there was no significant difference in medical research ethical training between "Professional Title". Regarding to the length of training time, Seniors spend 30 days which was significantly more than those juniors and middles with 3-5 days per year $(\mathrm{P}<0.0001)$. For training location, most training courses were held in Shenzhen. Noticeably, there were significant differences in training location outside of Shenzhen and overseas between "Professional title" as well $(\mathrm{P}<0.0001)$.

Compared with technicians and nurses, medical doctors and research faculty only had more chances for statistics training $(\mathrm{P}<0.0001)$.There was no significant difference in clinical research knowledge and skills, and research ethical training by job category. In addition, there was no difference between the roles of those who conducted the clinical research regarding to attending regulatory and ethical training. The percent of Principal Investigators and those who involved in clinical research process were only $45.1 \%(n=83), 41.6 \%(n=187)$.

\subsection{Training Needs}

According to the Training subjects and contents preference order, "Protocol design and development" was ranked first (60.3\%), followed by "Data analysis and summary" (49.1\%), "Project management and quality control" (48.0\%) and 
"Regulation and Research ethics"(33.5\%). Training subjects and contents preference order by Job category are listed in Table 3.There was significant difference in other key contents except ethics training.

Table 3. Training subjects and contents preference order by Job category

\begin{tabular}{lllllll}
\hline Subjects and contents & $\begin{array}{l}\text { Junior } \\
(\mathrm{N}=284)\end{array}$ & $\begin{array}{l}\text { Middle } \\
(\mathrm{N}=223)\end{array}$ & $\begin{array}{l}\text { Senior } \\
(\mathrm{N}=137)\end{array}$ & $\begin{array}{l}\text { Total } \\
(\mathrm{N}=644)\end{array}$ & P-value \\
\hline $\begin{array}{l}\text { Protocol design and } \\
\text { development }\end{array}$ & $137(48.2)$ & $152(68.2)$ & $99(72.3)$ & $388(60.3)$ & $<.0001$ \\
$\begin{array}{l}\text { Data analysis and summary } \\
\begin{array}{l}\text { Regulation and Research } \\
\text { ethics }\end{array}\end{array}$ & $128(45.1)$ & $109(48.9)$ & $79(57.7)$ & $316(49.1)$ & 0.0531 \\
$\begin{array}{l}\text { Project management and } \\
\text { quality control }\end{array}$ & $142(50.0)$ & $111(49.8)$ & $56(40.9)$ & $309(48.0)$ & 0.1718 \\
\hline
\end{tabular}

Nearly $72.0 \%$ of the respondents preferred to take the training course in working hospitals as the training type. Regarding to the obtaining methods and resources of clinical research knowledge, there was a statistically significant difference between job categories. The proportion of nurses, technicians, medical doctors and research faculty who preferred to obtain training outside of Shenzhen was $58.4 \%, 61.2 \%$ and $72.7 \%$ rather than in hospitals $(76.9 \%, 75.7 \%$ and $65.7 \%)$, respectively.

\subsection{Open-ended Questions}

Regarding to the elements of the course they most valued, and areas requiring improvement. The most prevalent theme arising from free-text comments was that the involvement of interactive sections would be highly beneficial for learning. For instance, "Theory and practice combination would be ideal to obtain training during conducting clinical research." and "Clinical research knowledge and skills should be taught systematically in courses rather than conferences." "On-line or distance training are preferred for those who are inconvenience by extended travel to attend a training site."

\section{Discussion}

It is essential to get access to ethical training and obtain certificates before conducting clinical research activities. However, less than $50 \%$ of the investigators have attended the research ethical training from our survey. Furthermore, regulatory and ethical contents is ranked last in demand and preference of training needs. This survey obviously indicated that ethical training availability and awareness was not sufficient. A similar situation was reported in another survey on training of clinical research nurses in China. Senior research nurses did not have adequate knowledge on informed consent (Shao et., al, 2015). Ethical knowledge is not adequately understood or valued. The ability of hospitals to conduct clinical research is at risk. Therefore, training on ethics aspects for health professionals should be offered in medical research as a prerequisite and strengthened in Shenzhen.

Regarding the subjects and contents preference order, the first ranked is "protocol design and developing" for medical/scientific research staff, which is the same as the survey of 1122 medical doctors from first -class hospitals in China (Zhang et., al 2015). However, it was shown that technicians and nurses were most willing to attend the "project management and quality control" training. This is in line with their possible role in the research project. Our survey showed that there were statistically significant more Seniors attending longer training for clinical research knowledge and skills, and statistics knowledge than juniors and middles. Wang reported that the needs were significantly different among nurses with different educational background and professional titles (Wang et., al, 2014).Thus, training course should be developed and designed according to the role. For instance, clinical research coordinators need focus on practical skills for complying with regulations and protocol.

Regarding the methods and resources related to clinical research knowledge, it was reported that $86.6 \%$ of the doctors have been trained for clinical research knowledge and skills by academic conference which is similar to that or our survey. Compared with technicians and nurses, medical doctors and research faculty have more chances for clinical research training since they are more likely to take part in academic symposiums or seminars. However, viable training and learning opportunities for junior fellows also should be considered. Modern concepts and skills of clinical research methodology, design and implementation features, and ethical regulations should be integrated as part of the professional continuing education activities.

The limitation of the survey was that the response rate was much lower than that we expected. We have concerns that the low response rate may not be representative. Due to the anonymous design, even the hospital management office did not know which individual failed to complete the survey. We only knew the total response rate in each hospital. Hospitals with low response rate sent a reminder to complete the survey. Thus, we could not analyze the cause and features between those who responded and those who did not. It seems that those who intend to become involved in clinical research would complete the survey. 
Clinical research training differs from basic science education. Research competency-based training programs should be developed and run by the research institutions. From the perspective of learners, both lectures and practical session such as case studies, interactive discussions and mentor ship should be preferably developed. Small group, structured discussions provided an environment conductive to learning for competency-based course (Zhang et., al, 2015).

\section{References}

Hu, Y. H., Huang, Y. Q., Ding, J., Liu, Y. L., Fan, D. S., Li, T. J., ... Ke, Y. (2011). Status of clinical research in China.The Lancet, 377(9760), 124-125. https://doi.org/10.1016/S0140-6736(11)60017-2

Shao, P., Gwenyth, R. W., Wang, J., Liu, X. W., Niu, W., \& Ni, C. P. (2015). Probe into study on training of clincial research nurses in China. Chinese nursing research, 29(6), 2026-2027. http://doi:10.3969/j.issn.10096493.2015.16.038

Wang, B. F., Chen, H. Y., Ma, J. H., Li, Y., Yu, H., Zhu, Y. F., \& Gu, Y. M. (2014). Research training needs among clinical nurses. Chinese nursing Management, 14(4), 423-426. http://doi:10.3969/j.issn.1672-1756.2014.04.02

Wang, C., \& Liu, Q. (2013). A turning point for clinical research in China? The Lancet, 382(9895), 835-836. https://doi.org/10.1016/S0140-6736(13)61804-8

Zhang, J. Y., Wang, X. Q., Shi, L., He, J. F., Bian, Y. L., \& Liu, C. Q. (2015). Investigation on Chinese clinician's perception of clinical trials. Chinese Journal Clinicians, 9(5), 881-885. https://doi.org/10.1007/s11606-007-0397-8

Zhang, Y., An, R., He, L. Y., Chen, L. N., Liu, S. Y., Cai, H. Y., ... \& Wang, S. C. (2010). Investigation and analysis on actuality and demands of scientific researchers in state clinical research facility of TCM. International Journal of traditional Chinese Medicine, 32(6), 523-524. http://doi:10.3760/cma.j.issn.1673-4246.2010.06.02

\section{Copyrights}

Copyright for this article is retained by the author(s), with first publication rights granted to the journal.

This is an open-access article distributed under the terms and conditions of the Creative Commons Attribution license which permits unrestricted use, distribution, and reproduction in any medium, provided the original work is properly cited. 